\title{
Energy Spaces, Dirichlet Forms and Capacities in a Nonlinear Setting
}

\section{Burkhard Claus ${ }^{1}$}

Received: 9 December 2019 / Accepted: 27 May 2021 /Published online: 18 June 2021

(C) The Author(s) 2021

\begin{abstract}
In this article we study lower semicontinuous, convex functionals on real Hilbert spaces. In the first part of the article we construct a Banach space that serves as the energy space for such functionals. In the second part we study nonlinear Dirichlet forms, as defined by Cipriani and Grillo, and show, as it is well known in the bilinear case, that the energy space of such forms is a lattice. We define a capacity and introduce the notion quasicontinuity associated with these forms and prove several results, which are well known in the bilinear case.
\end{abstract}

Keywords Nonlinear Semigroup Theory · Nonlinear Dirichlet form · Capacity · Quasicontinuity

Mathematics Subject Classification (2010) 31C45 - 31C25 - 35K55 - 47H20 - 58E30

\section{Introduction}

The theory of gradients and subgradients of convex functions on a Hilbert space, as presented in [1,2] or [3], can be seen as a nonlinear counterpart to the theory of symmetric bilinear forms.

In the setting of Lions, one is given a Gelfand triple $V \hookrightarrow H \hookrightarrow V^{\prime}$ and a convex, differentiable function $\mathcal{E}: V \rightarrow \mathbb{R}$. Then, the gradient is a nonlinear operator from $V$ to $V^{\prime}$. In this situation, the Banach space $V$ is sometimes called the energy space.

In the setting of Brezis, where $\mathcal{E}: H \rightarrow[0, \infty]$ is merely a convex, lower semicontinuous function on the Hilbert space $H$, such an energy space is not explicitly given. One goal of this article is to show that nevertheless a Banach space serving as an energy space can be constructed naturally, and therefore to partly unify the approaches of Lions and Brezis. In [4] the authors equipped the effective domain of the functional with a metric but here we are interested in a linear space.

Burkhard Claus

burkhard.claus@tu-dresden.de

1 Department of Mathematics, TU Dresden, Dresden, Germany 
In the case of quadratic forms, this energy space is the domain of the bilinear form equipped with the usual Hilbert space structure, and in the general case the energy space is a Banach space. For example the energy space of the $p$-Laplace operator on an open domain $X \subseteq \mathbb{R}^{n}$ with Neumann boundary conditions is the Sobolev space

$$
W_{p, 2}^{1}(X)=\left\{u \in L^{2}(X, m) \mid \nabla u \in L^{p}(X, m)\right\} .
$$

The second goal of this article is to study these energy spaces, when the functional is a nonlinear Dirichlet form. These forms were introduced by Cipriani and Grillo in [5] as convex, lower semicontinuous functionals on an $L^{2}$ space which generate order preserving and $L^{\infty}$ contractive semigroups of nonlinear operators. Furthermore, they showed, using earlier results form Barthelemy [6] as well as Bénilan and Picard [7], that this definition is equivalent to the intrinsic Definition 4.1 which we use in this paper.

In the theory of bilinear Dirichlet forms the Dirichlet spaces and the capacity are important building blocks of the vast theory of these forms and the semigroups as well as the Markov processes they generate [8-10]. We show that the Dirichlet space of a nonlinear Dirichlet form is a lattice and, under some assumptions, the lattice operation are continuous. We define a capacity and quasicontinuous functions and show that many of the results from the bilinear world still can be transferred to our setting.

In a forthcoming paper we want to use the results presented here to investigate boundary conditions and perturbations of Dirichlet forms.

We point out that in the article $[11,12]$ a capacity is defined in the nonlinear setting, too. The assumption $(H 0)$ in both articles assumes that the Dirichlet space exists and that the functional is $p$-homogeneous. It turns out that both assumptions are not necessary to define a capacity and we give an explicit way to construct the Dirichlet space. In addition, non homogeneous examples like the energy of the $\infty$-Laplacian are also covered in our approach. Other examples include the $p$ or $p(x)$-Laplacian on subsets of $\mathbb{R}^{d}$ or arbitrary Riemannian manifolds, fractional versions of these operators and sums thereof.

\section{Energy Spaces of Symmetric Functionals}

In the following $H$ is a real Hilbert space and $\mathcal{E}: H \rightarrow[0, \infty]$ always denotes a convex and lower semicontinuous functional. We define the effective domain

$$
\operatorname{dom} \mathcal{E}=\{x \in H \mid \mathcal{E}(x)<\infty\},
$$

and call $\mathcal{E}$

(i) symmetric, if $\mathcal{E}(0)=0$ and $\mathcal{E}(-x)=\mathcal{E}(x)$ for all $x \in L^{2}(X, m)$,

(ii) quasilinear, if $\operatorname{dom} \mathcal{E}$ is a linear subspace of $H$.

Remark 2.1 For a convex functional $\mathcal{E}: H \rightarrow[0, \infty]$, the condition $\mathcal{E}(-x)=\mathcal{E}(x)$ already implies that 0 is a global minimizer of $\mathcal{E}$. In the study of lower semicontinuous functionals in the context of partial differential equations and calculus of variations we are mostly interested in the minimizers and not the minimum itself. So without loss of generality we can assume that $\mathcal{E}(0)=0$.

We now construct a Banach space associated with the convex, lower semicontinuous functional $\mathcal{E}$. This construction in based on the ideas of modular spaces used in the construction of Musielak-Orlicz space and variable exponent Lebesgue space. For reference of this procedure see [13] or [14]. 
Let

$$
\mathcal{E}_{1}(x):=\|x\|_{H}^{2}+\mathcal{E}(x)
$$

and $\|\cdot\|_{\mathfrak{D}}: H \rightarrow[0, \infty]$ be defined as

$$
\|x\|_{\mathfrak{D}}:=\inf \left\{\lambda>0 \mid \mathcal{E}_{1}\left(\frac{x}{\lambda}\right) \leq 1\right\} .
$$

We define the energy space of $\mathcal{E}$ by

$$
\mathfrak{D}:=\left\{x \in H \mid\|x\|_{\mathfrak{D}}<\infty\right\}
$$

Lemma 2.2 Let $\mathcal{E}: H \rightarrow \mathbb{R} \cup\{\infty\}$ be a symmetric and convex functional on some Hilbert space. Then

$$
\mathfrak{D}=\left\{x \in H \mid \exists \lambda>0: \mathcal{E}_{1}(\lambda x)<\infty\right\}
$$

and $\mathfrak{D}=\operatorname{span}(\operatorname{dom} \mathcal{E})$.

Proof Let $x \in \mathfrak{D}$, that is there exists a $\lambda>0$ such that

$$
\mathcal{E}_{1}\left(\frac{x}{\lambda}\right) \leq 1
$$

Hence, $x \in\left\{x \in H \mid \exists \lambda>0: \mathcal{E}_{1}(\lambda x)<\infty\right\}$. On the other hand, if $x \in\{x \in H \mid \exists \lambda>$ $\left.0: \mathcal{E}_{1}(\lambda x)<\infty\right\}$ there is a $\lambda>0$ such that $\mathcal{E}_{1}(\lambda x)=C<\infty$. Either $C \leq 1$, then the statement follows directly or $C>1$. In the case $C>1$ we have

$$
\begin{aligned}
\mathcal{E}_{1}\left(\frac{\lambda}{C} x\right) & \leq \mathcal{E}_{1}\left(\frac{\lambda}{C} x+\left(1-\frac{1}{C}\right) 0\right) \\
& \leq \frac{1}{C} \mathcal{E}_{1}(\lambda x)+0 \\
& =1
\end{aligned}
$$

Hence, $x \in \mathfrak{D}$. The second part of the statement follows directly from the first.

Theorem 2.3 Let $\mathcal{E}: H \rightarrow[0, \infty]$ be a symmetric, convex, lower semicontinuous functional on $H$. Then the space $\left(\mathfrak{D},\|\cdot\|_{\mathfrak{D}}\right)$ is a Banach space. In addition, the embedding $i: \mathfrak{D} \rightarrow H$ is continuous and $\|\cdot\|_{\mathfrak{D}}$ is lower semicontinuous on $H$.

Proof Observe that for all $x \in H$ we have

$$
\|x\|_{H}=\inf \left\{\lambda>0:\left\|\frac{x}{\lambda}\right\|_{H}^{2} \leq 1\right\} \leq \inf \left\{\lambda>0:\left\|\frac{x}{\lambda}\right\|_{H}^{2}+\mathcal{E}\left(\frac{x}{\lambda}\right) \leq 1\right\}=\|x\|_{\mathfrak{D}} .
$$

Since $\|\cdot\|_{H}$ is a norm, this yields $\|x\|_{\mathfrak{D}}=0$ if and only if $x=0$.

To show homogeneity, let $\mu>0$. Then,

$$
\begin{aligned}
\|\mu x\|_{\mathfrak{D}} & =\inf \left\{\lambda>0 \mid \mathcal{E}_{1}\left(\frac{\mu x}{\lambda}\right) \leq 1\right\} \\
& =\inf \left\{\lambda \mu>0 \mid \mathcal{E}_{1}\left(\frac{\mu x}{\mu \lambda}\right) \leq 1\right\} \\
& =\inf \left\{\lambda \mu>0 \mid \mathcal{E}_{1}\left(\frac{x}{\lambda}\right) \leq 1\right\} \\
& =\mu \inf \left\{\lambda>0 \mid \mathcal{E}_{1}\left(\frac{x}{\lambda}\right) \leq 1\right\} \\
& =\mu\|x\|_{\mathfrak{D}} .
\end{aligned}
$$


Homogeneity for $\mu<0$ follows, since $\mathcal{E}_{1}(x)=\mathcal{E}_{1}(-x)$ which implies $\|-x\|_{\mathfrak{D}}=\|x\|_{\mathfrak{D}}$.

For the triangle inequality, let $x, y \in \mathfrak{D}$ and $a>\|x\|_{\mathfrak{D}}, b>\|y\|_{\mathfrak{D}}$. Then

$$
\begin{aligned}
\mathcal{E}_{1}\left(\frac{x+y}{a+b}\right) & =\mathcal{E}_{1}\left(\frac{a}{a+b} \frac{x}{a}+\frac{b}{a+b} \frac{y}{b}\right) \\
& \leq \frac{a}{a+b} \mathcal{E}_{1}\left(\frac{x}{a}\right)+\frac{b}{a+b} \mathcal{E}_{1}\left(\frac{y}{b}\right) \\
& \leq 1 .
\end{aligned}
$$

Hence

$$
\|x+y\|_{\mathfrak{D}} \leq a+b .
$$

taking the infimum over all possible $a$ and $b$ yields

$$
\|x+y\|_{\mathfrak{D}} \leq\|x\|_{\mathfrak{D}}+\|y\|_{\mathfrak{D}} .
$$

Thus $\|\cdot\|_{\mathfrak{D}}$ is a norm. Since we already showed that

$$
\|x\|_{H} \leq\|x\|_{\mathfrak{D}},
$$

we know that the embedding $\iota: \mathfrak{D} \rightarrow H$ is continuous.

It is easy to see, that $\mathcal{E}_{1}(x) \leq 1$ implies $\|x\|_{\mathfrak{D}} \leq 1$. On the other hand, if $\|x\|_{\mathfrak{D}} \leq 1$, then

$$
\mathcal{E}_{1}\left(\frac{x}{\lambda}\right) \leq 1
$$

for every $\lambda \geq 1$. Since $\mathcal{E}_{1}$ is lower semicontinuous on $L^{2}(X, m)$, taking the limit as $\lambda \searrow 1$ yields $\mathcal{E}_{1}(x) \leq 1$. Thus,

$$
\left\{x \in H \mid \mathcal{E}_{1}(x) \leq 1\right\}=\left\{x \in H \mid\|x\|_{\mathfrak{D}} \leq 1\right\} .
$$

Since $\mathcal{E}_{1}$ is lower semicontinous, the set

$$
B_{1}=\left\{x \mid\|x\|_{\mathfrak{D}} \leq 1\right\}
$$

is closed in $H$. Let $\mu \geq 0$. Then

$$
\left\{x \mid\|x\|_{\mathfrak{D}} \leq \mu\right\}=\mu B_{1}
$$

is also closed. Therefore $\|\cdot\|_{\mathfrak{D}}$ is lower semicontinuous on $\mathrm{H}$.

Let $\left(x_{n}\right)_{n}$ be a Cauchy sequence in $\mathfrak{D}$. Then $\left(x_{n}\right)_{n}$ is a Cauchy sequence in $H$. Since $H$ is complete, there is an $x \in H$ with $x_{n} \rightarrow_{H} x$ and, since the norm $\|\cdot\|_{\mathfrak{D}}$ is lower semicontinuous on $L^{2}(X, m)$ and Cauchy sequences are bounded,

$$
\|x\|_{\mathfrak{D}} \leq \liminf _{m \rightarrow \infty}\left\|x_{m}\right\|_{\mathfrak{D}}<\infty .
$$

Hence $x \in \mathfrak{D}$. Let $\epsilon>0$. We choose $N \in \mathbb{N}$ such that for every $n, m \geq N$

$$
\left\|x_{n}-x_{m}\right\|_{\mathfrak{D}}<\epsilon .
$$

Thus, the lower semicontinuity of the norm yields

$$
\left\|x_{n}-x\right\|_{\mathfrak{D}} \leq \liminf _{m \rightarrow \infty}\left\|x_{n}-x_{m}\right\|_{\mathfrak{D}}<\epsilon
$$

for every $n \geq N$. Therefore, $x_{n} \rightarrow \mathfrak{D} x$. Thus, $\mathfrak{D}$ is complete.

Let $\alpha>0$,

$$
\|x\|_{\mathfrak{D}, \alpha}=\inf \left\{\lambda>0: \mathcal{E}_{1}\left(\frac{x}{\lambda}\right) \leq \alpha\right\} .
$$


and

$$
|x|_{\mathfrak{D}}=\inf \left\{\lambda>0: \mathcal{E}\left(\frac{x}{\lambda}\right) \leq 1\right\} .
$$

Similar arguments as in the first part of the previous proof show, that $\|\cdot\|_{\mathfrak{D}, \alpha}$ are norms for every $\alpha>0$ and $|\cdot| \mathfrak{D}$ is a seminorm.

Theorem 2.4 For every $\alpha>0$, the norms $\|\cdot\|_{\mathfrak{D}},\|\cdot\|_{\mathfrak{D}, \alpha}$ and $\|\cdot\|_{H}+|\cdot|_{\mathfrak{D}}$ are equivalent norms on $\mathfrak{D}$.

Proof At first, we can use exactly the same proof as for $\|\cdot\|_{\mathfrak{D}}$ to show that $\mathfrak{D}$ together with $\|\cdot\|_{\mathfrak{D}, \alpha}$ is a Banach space. Let $0<\alpha_{1}<\alpha_{2}$. Then

$$
\|x\|_{\mathfrak{D}, \alpha_{2}} \leq\|x\|_{\mathfrak{D}, \alpha_{1}},
$$

for every $x \in \mathfrak{D}$, since $\|x\|_{\mathfrak{D}, \alpha_{2}}$ takes the infimum over a larger set. The open mapping theorem implies that comparable complete norms are equivalent. Thus, there is a $C>0$ such that

$$
\|x\|_{\mathfrak{D}, \alpha_{1}} \leq C\|x\|_{\mathfrak{D}, \alpha_{2}}
$$

for every $x \in \mathfrak{D}$.

For the second part, let $x \in \mathfrak{D}$. Obviously

$$
\|x\|_{H} \leq\|x\|_{\mathfrak{D}}
$$

and

$$
|x|_{\mathfrak{D}} \leq\|x\|_{\mathfrak{D}} .
$$

Hence

$$
\|x\|_{H}+|x|_{\mathfrak{D}} \leq 2\|x\|_{\mathfrak{D}} .
$$

On the other hand, let $\lambda_{1}, \lambda_{2}$ such that $\|x\|_{H} \leq \lambda_{1}$ and $|x|_{\mathfrak{D}} \leq \lambda_{2}$. Then

$$
\left\|\frac{x}{\lambda_{1}}\right\|_{H} \leq 1
$$

and

$$
\mathcal{E}\left(\frac{x}{\lambda_{2}}\right) \leq 1
$$

Hence,

$$
\mathcal{E}_{1}\left(\frac{x}{\lambda_{1}+\lambda_{2}}\right) \leq 2
$$

Thus,

$$
\|x\|_{\mathfrak{D}, 2} \leq \lambda_{1}+\lambda_{2} .
$$

Taking the infimum over all possible $\lambda_{1}$ and $\lambda_{2}$ yields

$$
\|x\|_{\mathfrak{D}, 2} \leq\|x\|_{H}+|x|_{\mathfrak{D}} .
$$

Since $\|x\|_{\mathfrak{D}, 2}$ and $\|x\|_{\mathfrak{D}}$ are equivalent, there is a $C>0$ such that

$$
C\|x\|_{\mathfrak{D}} \leq\|x\|_{H}+|x|_{\mathfrak{D}} \leq 2\|x\|_{\mathfrak{D}} .
$$

Hence all the norms are equivalent. 
Theorem 2.5 Let $\mathcal{E}$ be a symmetric, convex and lower semicontinuous functional on $H$. Then $\mathfrak{D}$ is a dual space.

Proof Kaijser proved in [15], that a Banach space $Y$ is a dual space if there is a set of continuous linear functionals $E$ on $Y$ that separates the points of $Y$ and the closed unit ball of $Y$ is compact in the weak topology generated by $E$.

Let $E=H^{\prime}=H$. By the Hahn-Banach theorem, $E$ separates the points of $H$. Hence, it separates the points of $\mathfrak{D}$. On the other hand the closed unit ball $B_{1}$ of $\mathfrak{D}$ is closed and bounded in $H$ as shown in Theorem 2.3. Therefore, it is compact in the weak* topology by Banach-Alaoglu. But weak and weak* topology coincide on reflexive spaces. Hence, $B_{1}$ is compact in the weak topology induced by $E$. Thus $\mathfrak{D}$ is a dual space.

Note that Kaijser also showed that the predual is then given by the weak* closure of span $E$ in $\mathfrak{D}^{\prime}$.

Theorem 2.6 Let $\mathcal{E}$ be a symmetric, convex and lower semicontinuous functional on $H$ and let $\left(x_{n}\right)_{n \in \mathbb{N}}$ be a sequence in $\mathfrak{D}$. Then $x_{n} \rightarrow 0$ in $\mathfrak{D}$ if and only if for all $\lambda>0: \mathcal{E}_{1}\left(\lambda x_{n}\right)$ $\rightarrow 0$.

Theorem 2.7 (Norm-modular unit ball property) Let $\mathcal{E}$ be a symmetric, convex and lower semicontinuous functional on $H$ and let $x \in \mathfrak{D}$.

(i) If $\|x\|_{\mathfrak{D}} \leq 1$, then $\mathcal{E}_{1}(x) \leq\|x\|_{D}$.

(ii) If $\|x\|_{\mathfrak{D}}>1$, then $\|x\|_{D} \leq \mathcal{E}_{1}(x)$.

(iii) $\|x\| \leq \mathcal{E}_{1}(x)+1$.

Proof For both theorems we omit the proof, see [14, Lemmas 2.1.9, 2.1.14 and Corollary $2.1 .15]$ instead. Note that the convexity and lower semicontinuity of $\mathcal{E}$ and $\mathcal{E}_{1}$ imply that $\mathcal{E}_{1}$ is left continuous and therefore a modular in the notation of this reference.

\section{Energy Spaces of non Symmetric Functionals}

Definition 3.1 Let $\mathcal{E}: H \rightarrow[0, \infty]$ be a lower semicontinuous, convex functional on a real Hilbert space $H$, such that $\mathcal{E}(0)=0$. We define the symmetric closure sym $\mathcal{E}$ of $\mathcal{E}$ by

$$
\operatorname{sym} \mathcal{E}(u)=\sup \left\{\mathcal{F}(u) \mid \mathcal{F} \text { lsc, convex and } \mathcal{F} \leq \mathcal{E}_{1}(\cdot), \mathcal{E}_{1}(-\cdot)\right\} \text {. }
$$

Lemma 3.2 Let $\mathcal{F}: H \rightarrow[0, \infty]$ be a functional on some Hilbert space $H$. Then

$$
\overline{\operatorname{Conv}(\text { epigraph }(\mathcal{F}))}=\operatorname{epigraph}(x \mapsto \sup \{\mathcal{G}(x) \mid \mathcal{G} \text { lsc, convex and } \mathcal{G} \leq \mathcal{F}\}) \text {. }
$$

Proof Note that the claim is trivial in the case $\mathcal{F}(x)=\infty$ for every $x \in H$. Therefore, let us assume that $\mathcal{F}$ is proper, that is there is a $x \in H$ such that $\mathcal{F}(x)<\infty$.

Since $\hat{\mathcal{F}}: x \mapsto \sup \{\mathcal{G}(x) \mid \mathcal{G}$ lsc, convex and $\mathcal{G} \leq \mathcal{F}\}$ is a convex and lower semicontinuous functional, epigraph $\hat{\mathcal{F}}$ is convex and closed. In addition, $\hat{\mathcal{F}} \leq \mathcal{F}$ implies

$$
\text { epigraph } \mathcal{F} \subseteq \text { epigraph } \hat{\mathcal{F}} \text {. }
$$


Therefore,

$$
\overline{\operatorname{Conv}(\text { epigraph }(\mathcal{F}))} \subseteq \operatorname{epigraph}(\hat{F}) .
$$

On the other Hand, let $M \subseteq H \times \mathbb{R}$ be a closed and convex set such that epigraph $(\mathcal{F}) \subseteq M$ and $(x, t) \in M$. Since $\mathcal{F}$ is proper, that is epigraph $\mathcal{F} \neq \emptyset$, and epigraph $\mathcal{F} \subseteq M$, there is a $x_{0} \in H$ and $t_{0} \in \mathbb{R}$ such that

$$
x_{0} \times\left[t_{0}, \infty\right) \subseteq M
$$

Let $h>0$ and let us define

$$
h_{n}=n h+t-t_{0} .
$$

Then $h_{n} \geq 0$ for every $n \in \mathbb{N}$ such that $n>\frac{t_{0}-t}{h}$. Since $M$ is convex and $\left(x_{0}, t_{0}+h_{n}\right) \in M$ for large $n$,

$$
\left(\left(1-\frac{1}{n}\right) x+\frac{1}{n} x_{0}, t+h\right)=\left(1-\frac{1}{n}\right)(x, t)+\frac{1}{n}\left(x_{0}, t_{0}+h_{n}\right) \in M
$$

for large $n$. Since $M$ is closed,

$$
(x, t+h) \in M
$$

and since $h>0$ was arbitrary, $x \times[t, \infty) \subseteq M$. Hence, $M$ is the epigraph of the functional

$$
\varphi(x)=\inf \{t \mid(x, t) \in M\} .
$$

Since $M=$ epigraph $\varphi$ is closed and convex, $\varphi$ is lower semicontinuous and convex. In addition, $\varphi(x) \leq \mathcal{F}(x)$ for every $x \in H$, since epigraph $\mathcal{F} \subseteq M$. Thus,

$$
\varphi(x) \leq \hat{\mathcal{F}}(x)
$$

for every $x \in H$ or, equivalently,

$$
\text { epigraph } \hat{\mathcal{F}} \subseteq \text { epigraph } \varphi=M .
$$

Taking the intersection of all closed and convex $M$ such that epigraph $(\mathcal{F}) \subseteq M$ yields

$$
\text { epigraph } \hat{\mathcal{F}} \subseteq \overline{\operatorname{Conv}(\text { epigraph }(\mathcal{F}))} \text {. }
$$

Hence, we showed

$$
\text { epigraph } \hat{\mathcal{F}}=\overline{\operatorname{Conv}(\text { epigraph }(\mathcal{F}))} \text {. }
$$

Theorem 3.3 Let $\mathcal{E}: H \rightarrow[0, \infty]$ be a lower semicontinuous, convex functional on a Hilbert $H$, such that $\mathcal{E}(0)=0$. Let $\mathfrak{D}$ denote the Banach space associated with sym $\mathcal{E}$. Then

$$
\mathfrak{D}=\operatorname{span} \operatorname{dom}(\mathcal{E})=\operatorname{span} \operatorname{dom}(\operatorname{sym} \mathcal{E}) .
$$

Moreover, any $f \in \operatorname{dom}(\operatorname{sym} \mathcal{E})$ can be written as $f=u-v$ for $u, v \in \operatorname{dom}(\mathcal{E})$.

Proof We need to show that $\operatorname{dom}(\operatorname{sym} \mathcal{E}) \subseteq \operatorname{span} \operatorname{dom}(\mathcal{E})$. For this purpose, let $f \in$ $\operatorname{dom}(\operatorname{sym} \mathcal{E})$. Then, there is a $t \geq 0 \operatorname{such}$ that $(f, t) \in \operatorname{epigraph}(\operatorname{sym} \mathcal{E})$.

By Lemma 3.2 and the fact that $\mathcal{E}_{1}$ and $\mathcal{E}_{1}(-\cdot)$ are convex, epigraph $(\operatorname{sym} \mathcal{E}$ ) is the closure of

$$
\operatorname{Conv}\left(\text { epigraph }\left(\mathcal{E}_{1}(\cdot)\right) \cup \operatorname{epigraph}\left(\mathcal{E}_{1}(-\cdot)\right)\right)
$$


Hence, there is a sequence $\left(f_{n}, t_{n}\right)_{n \in \mathbb{N}}$ in $\operatorname{Conv}\left(\right.$ epigraph $\left(\mathcal{E}_{1}(\cdot) \cup\right.$ epigraph $\left.\left(\mathcal{E}_{1}(-\cdot)\right)\right)$ such that $\left(f_{n}, t_{n}\right) \rightarrow(f, t)$ in $H \times \mathbb{R}$. By definition of the convex hull, there are $\left(u_{n}, s_{n}\right),\left(v_{n}, r_{n}\right) \in$ epigraph $\mathcal{E}_{1}$ and $\lambda_{n} \in[0,1]$ such that

$$
\left(f_{n}, t_{n}\right)=\lambda_{n}\left(u_{n}, s_{n}\right)+\left(1-\lambda_{n}\right)\left(-v_{n}, r_{n}\right) .
$$

After choosing a subsequence, we may assume that $\lambda_{n} \rightarrow \lambda \in[0,1]$. Now, let us assume $\lambda \neq 0$. Since $r_{n}$ and $s_{n}$ are positive,

$$
0 \leq \lambda_{n} s_{n} \leq t_{n} \rightarrow t .
$$

Hence, $\left(s_{n}\right)_{n \in \mathbb{N}}$ is bounded since $\lambda \neq 0$. Again, after choosing a subsequence if necessary, there is a $s \in \mathbb{R}$ such that $s_{n} \rightarrow s$. By the definition of the epigraph, we know that

$$
\mathcal{E}\left(u_{n}\right)+\left\|u_{n}\right\|_{H}^{2} \leq s_{n} \rightarrow s .
$$

Hence, $\left(u_{n}\right)_{n \in \mathbb{N}}$ is bounded in $H$. Thus there is a subsequence, again denoted by $\left(u_{n}\right)_{n \in \mathbb{N}}$, such that $u_{n} \rightarrow u$. Since $\mathcal{E}$ is lower semicontinuous with respect to the weak topology on $H, u \in \operatorname{dom} \mathcal{E}$. On the other hand,

$$
f_{n}=\lambda_{n} u_{n}-\left(1-\lambda_{n}\right) v_{n},
$$

and $f_{n} \rightarrow f, u_{n} \rightarrow u$ implies $\left(1-\lambda_{n}\right) v_{n} \rightarrow v$. Hence,

$$
\begin{aligned}
\mathcal{E}(v) & \leq \liminf _{n \rightarrow \infty} \mathcal{E}\left(\left(1-\lambda_{n}\right) v_{n}\right) \\
& \leq \liminf _{n \rightarrow \infty}\left(1-\lambda_{n}\right) \mathcal{E}\left(v_{n}\right) \\
& \leq \liminf _{n \rightarrow \infty}\left(1-\lambda_{n}\right) r_{n} \\
& =\liminf _{n \rightarrow \infty}\left(t_{n}-\lambda_{n} s_{n}\right) \\
& =t-\lambda s \\
<\infty &
\end{aligned}
$$

Thus, $v \in \operatorname{dom}(\mathcal{E})$ and $f=\lambda u-v$. Hence, $f \in \operatorname{span} \operatorname{dom}(\mathcal{E})$.

If $\lambda=0$ then $1-\lambda \neq 0$ and switching $r$ and $s$ as well as $u$ and $v$ in the previous argument yields the conclusion.

Definition 3.4 Let $\mathcal{E}: H \rightarrow[0, \infty]$ be a lower semicontinuous, convex functional on a Hilbert $H$, such that $\mathcal{E}(0)=0$. We call the space $\mathfrak{D}$ associated with sym $\mathcal{E}$ the energy space of $\mathcal{E}$.

Remark 3.5 If $\mathcal{E}$ is already symmetric, then $\operatorname{sym} \mathcal{E}=\mathcal{E}_{1}$ and both definitions of $\mathfrak{D}$ coincide.

Remark 3.6 In this article we always start with a Hilbert space H. For the results up to this point this is in fact not necessary. Similar results hold in the case of reflexive Banach spaces like for example $L^{p}$ for $1<p<\infty$. It might be more natural in some applications, for example if $\mathcal{E}$ itself is $p$-homogeneous, to define

$$
\mathcal{E}_{1}(\cdot)=\|\cdot\|_{L^{p}}^{p}+\mathcal{E}(\cdot)
$$

which leads again to a $p$-homogeneous functional.

Remark 3.7 If 0 is not a global minimizer of $\mathcal{E}$, but if nevertheless $\mathcal{E}$ possesses a global minimizer, then we can shift the functional $\mathcal{E}$ and still define an energy space. This shifted functional is still a Dirichlet form if $\mathcal{E}$ is a Dirichlet form. But one looses the property $\operatorname{dom} \mathcal{E} \subseteq \mathfrak{D}$ if one defines $\mathfrak{D}$ by using the shifted functional. 
Remark 3.8 We also assume that $\mathcal{E}$ is convex. But in fact we only need that $\mathcal{E}_{1}$ is convex, or more general that

$$
\mathcal{E}_{\alpha}(x)=\|x\|_{H}+\alpha \mathcal{E}(x)
$$

is convex for some $\alpha>0$. Then $\mathcal{E}_{\alpha}$ generates an energy space and the norms are equivalent for any $\alpha$ such that $\mathcal{E}_{\alpha}$ is convex. To prove this, let $\alpha<\beta$ such that $\mathcal{E}_{\alpha}$ and $\mathcal{E}_{\beta}$ are convex. Then $\mathcal{E}_{\alpha}$ resp. $\mathcal{E}_{\beta}$ generate an energy space $\mathfrak{D}_{\alpha}$ resp. $\mathfrak{D}_{\beta}$. By Theorem 5.4, the norms $\|\cdot\|_{\mathfrak{D}_{\alpha}}$ and $\|\cdot\|_{\mathfrak{D}_{\beta}}$ are equivalent to $\|\cdot\|_{H}+|\cdot|_{\mathfrak{D}_{\alpha}}$ and $\|\cdot\|_{H}+|\cdot|_{\mathfrak{D}_{\beta}}$. Obviously

$$
|x|_{\mathfrak{D}_{\alpha}} \leq|x|_{\mathfrak{D}_{\beta}}
$$

by definition. On the other hand,

$$
\begin{aligned}
|x|_{\mathfrak{D}_{\beta}} & =\inf \left\{\lambda>0: \beta \mathcal{E}\left(\frac{x}{\lambda}\right) \leq 1\right\} \\
& =\inf \left\{\lambda>0: \alpha \frac{\beta}{\alpha} \mathcal{E}\left(\frac{x}{\lambda}\right) \leq 1\right\} \\
& \leq \inf \left\{\lambda>0: \alpha \mathcal{E}\left(\frac{\beta x}{\alpha \lambda}\right) \leq 1\right\} \\
& =\inf \left\{\frac{\beta}{\alpha} \lambda>0: \alpha \mathcal{E}\left(\frac{x}{\lambda}\right) \leq 1\right\} \\
& =\frac{\beta}{\alpha}|x|_{\mathfrak{D}_{\alpha}} .
\end{aligned}
$$

Hence, the norms on $\mathfrak{D}_{\alpha}$ and $\mathfrak{D}_{\beta}$ are equivalent. Thus the energy space does not depend on the choice of $\alpha$ and we can define an energy space for any functional $\mathcal{E}$ such that

$$
\mathcal{E}_{\alpha}(x)=\|x\|_{H}+\alpha \mathcal{E}(x)
$$

is convex. Such functionals $\mathcal{E}$ are called $\omega$-semiconvex, where $\omega=\frac{1}{\alpha}$.

Taking the previous remarks into account we can define an energy space for any lower semicontinuous, semiconvex functional $\mathcal{E}$ on any reflexive Banach space.

\section{Dirichlet Forms and Dirichlet Spaces}

Let $X$ be a countably generated Borel space and $m$ a $\sigma$-finite Borel measure such that $\operatorname{supp}(m)=X$. The following definition was first introduced by [5].

Definition 4.1 Let $\mathcal{E}: L^{2}(X, m) \rightarrow[0, \infty]$ be a convex and lower semicontinuous functional with dense effective domain. We call $\mathcal{E}$ a Dirichlet form if

$$
\mathcal{E}(u \wedge v)+\mathcal{E}(u \vee v) \leq \mathcal{E}(u)+\mathcal{E}(v)
$$

and

$$
\begin{aligned}
\mathcal{E}(v+ & \left.\frac{1}{2}\left((u-v+\alpha)_{+}-(u-v-\alpha)_{-}\right)\right) \\
& +\mathcal{E}\left(u-\frac{1}{2}\left((u-v+\alpha)_{+}-(u-v-\alpha)_{-}\right)\right) \leq \mathcal{E}(u)+\mathcal{E}(v)
\end{aligned}
$$

for every $u, v \in L^{2}(X, m), \alpha>0$.

If $\mathcal{E}(0)=0$ we call the energy space $\mathfrak{D}$ of $\mathcal{E}$ the Dirichlet space. 
Remark 4.2 It is well known, that a convex, lower semicontinuous functional with dense domain generates a semigroup of nonlinear contractions. In the theory of bilinear forms, Dirichlet forms are exactly those forms, which generate sub-Markovian semigroups, that is order-preserving and $L^{\infty}$-contractive semigroups. The two conditions in the previous definition hold if and only if the semigroup generated by $\mathcal{E}$ is sub-Markovian.

Remark 4.3 If $a$ is a bilinear Dirichlet form, then

$$
\mathcal{E}(u)= \begin{cases}\frac{1}{2} a(u, u) & \text { if } x \in D(a) \\ \infty & \text { otherwise }\end{cases}
$$

is a Dirichlet form in the sense of the previous definition and the Dirichlet space $\mathfrak{D}$ and the classical Dirichlet space, that is the form domain $D(a)$ together with the scalar product $a_{1}$, are isomorphic as topological vector spaces. Furthermore, there is a scalar product on $\mathfrak{D}$ which induces the norm $\|\cdot\|_{\mathfrak{D}}$.

Remark 4.4 Let $\mathcal{E}$ be a symmetric Dirichlet form and $u \in L^{2}(X, m)$. Then inequality (2) in the definition of a Dirichlet form and the symmetry of $\mathcal{E}$ directly yield

$$
\mathcal{E}(|u|) \leq \mathcal{E}(u) \text { and } \mathcal{E}\left(u^{ \pm}\right) \leq \mathcal{E}(u) .
$$

This implies

$$
\||u|\|_{\mathfrak{D}} \leq\|u\|_{\mathfrak{D}} \text { and }\left\|u^{ \pm}\right\| \leq\|u\|
$$

and

$$
\left.|| u\right|_{\mathfrak{D}} \leq|u|_{\mathfrak{D}} \text { and }\left|u^{ \pm}\right| \leq|u| .
$$

Definition 4.5 We call $p \in W^{1, \infty}(\mathbb{R})$ a normal contraction if $0 \leq p^{\prime} \leq 1$ and $p(0)=0$.

Remark 4.6 Note that any such normal contraction $p$ induces an operator $T_{p}: L^{2}(X, m) \rightarrow$ $L^{2}(X, m)$ defined by

$$
\left(T_{p} u\right)(x)=p(u(x)) .
$$

We do not distinguish between $p$ and $T_{p}$ and denote both objects by $p$.

Theorem 4.7 (Beurling-Deny Criterion) Let $\mathcal{E}: L^{2}(X, m) \rightarrow[0, \infty]$ be a convex, lower semicontinuous functional. Then $\mathcal{E}$ is a Dirichlet form if and only if

$$
\mathcal{E}(u-p(u-v))+\mathcal{E}(v+p(u-v)) \leq \mathcal{E}(u)+\mathcal{E}(v)
$$

for every $u, v \in L^{2}(X, m)$ and every normal contraction $p$.

The proof follows from [7, Proposition 1.2, Lemma 7.1, Proposition 7.2] and [16, Corollary 2.1].

Theorem 4.8 Let $\mathcal{E}$ be a Dirichlet form on $L^{2}(X, m)$ such that $\mathcal{E}(0)=0$. Then the energy space $\mathfrak{D}$ associated with $\operatorname{sym} \mathcal{E}$ is a Riesz subspace of $L^{2}(X, m)$. That is, for $u, v \in \mathfrak{D}$ we have

$$
u \wedge v, u \vee v \in \mathfrak{D}
$$


Proof Let $g \in \mathfrak{D}$. Since

$$
\mathfrak{D}=\left\{f \in L^{2}(X, m) \mid \text { there is } \lambda>0 \text { such that } \lambda f \in \operatorname{dom}(\operatorname{sym} \mathcal{E})\right\},
$$

there is a $\lambda>0$ and a function $f \in \operatorname{dom}(\operatorname{sym} \mathcal{E})$ such that $g=\lambda f$. We showed in Theorem 3.3 , that there are $u, v \in \operatorname{dom}(\mathcal{E})$ such that $f=u-v$. Hence,

$$
\begin{aligned}
g \vee 0 & =(\lambda u-\lambda v) \vee 0 \\
& =\lambda(u-v) \vee 0 \\
& =\lambda(u \vee v)-\lambda v .
\end{aligned}
$$

Since $u \vee v, v \in \operatorname{dom}(\mathcal{E}), g \vee 0 \in \operatorname{span} \operatorname{dom}(\mathcal{E})=\mathfrak{D}$. The same argument works for $g \wedge 0$. This implies the claim, since

$$
x \wedge y=(x-y) \wedge 0+y .
$$

Theorem 4.9 Let $\mathcal{E}$ be a symmetric Dirichlet form on $L^{2}(X, m)$. Then

$$
\|u \wedge v\|_{\mathfrak{D}} \leq\|u\|_{\mathfrak{D}}+\|v\|_{\mathfrak{D}}
$$

for every $u, v \in \mathfrak{D}$.

Proof Let us assume the contrary. Then, there are $u, v \in \mathfrak{D}$ such that

$$
\|u \wedge v\|_{\mathfrak{D}}>\|u\|_{\mathfrak{D}}+\|v\|_{\mathfrak{D}} .
$$

Note that, either $u$ or $v$ is non zero. Therefore, we can choose a $\lambda \in\left(\frac{1}{\|u \wedge v\|_{\mathfrak{D}}}, \frac{1}{\|u\|_{\mathfrak{D}}+\|v\|_{\mathfrak{D}}}\right)$. This implies $\lambda\|u \wedge v\|_{\mathfrak{D}}>1$ and $\|\lambda u\|_{\mathfrak{D}},\|\lambda v\|_{\mathfrak{D}}<1$. Hence, by Theorem 2.7,

$$
\mathcal{E}_{1}(\lambda(u \wedge v)) \geq \lambda\|u \wedge v\|_{\mathfrak{D}}>\|\lambda u\|_{\mathfrak{D}}+\|\lambda v\|_{\mathfrak{D}} \geq \mathcal{E}_{1}(\lambda u)+\mathcal{E}_{1}(\lambda v) .
$$

Since $\mathcal{E}_{1}$ is a Dirichlet form, the previous inequality is a contradiction.

One could hope that the following even stronger inequality holds

$$
\|u \wedge v\|_{\mathfrak{D}}+\|u \vee v\|_{\mathfrak{D}} \leq\|u\|_{\mathfrak{D}}+\|v\|_{\mathfrak{D}} .
$$

But this is false, as the next example illustrates.

Example 4.10 Let $X=[0,1]$ and $m$ the Lebesgue measure. We consider the functional $\mathcal{E}: L^{2}(X, m) \rightarrow[0, \infty]$ given by

$$
\mathcal{E}(u)=\int_{X} \chi_{[0,1]}(|u(x)|) d x,
$$

where $\chi_{A}: \mathbb{R} \rightarrow\{0, \infty\}$ is given by

$$
\chi_{A}(x)= \begin{cases}0 & \text { if } x \in A, \\ \infty & \text { else }\end{cases}
$$

The functional $\mathcal{E}$ is lower semicontinuous, by Fatou's Lemma. Additionally, it is convex, since $\chi_{[0,1]}$ is convex and it is easy to verify that $\mathcal{E}$ satisfies the projection inequalities of Definition 4.1. Hence, it is a symmetric Dirichlet form. The norm $\|\cdot\|_{\mathfrak{D}}$ is induced by the functional

$$
\mathcal{E}_{1}(u)=\|u\|_{2}^{2}+\mathcal{E}(u) .
$$


By definition, the Dirichlet space $\mathfrak{D}$ is given by all elements $u \in L^{2}(X, m)$ such that there is a $\lambda>0$ satisfying

$$
\mathcal{E}\left(\lambda^{-1} u\right)=\int_{X} \chi_{[0,1]}\left(\left|\lambda^{-1} u(x)\right|\right) d x \leq 1,
$$

which is equivalent to

$$
\chi_{[0,1]}\left(\left|\lambda^{-1} u(x)\right|\right)=0
$$

almost everywhere, or in other words

$$
|u(x)| \leq \lambda \text { for almost all } x \in X
$$

Thus, $u \in \mathfrak{D}$ if and only if $u \in L^{\infty}(X, m)$. Analogously the norm is given by

$$
\|u\|_{\mathfrak{D}}=\max \left\{\|u\|_{2},\|u\|_{\infty}\right\} .
$$

Since $([0,1], m)$ is a probability space, the norm $\|\cdot\|_{\mathfrak{D}}$ is equal to $\|\cdot\|_{\infty}$. Now let

$$
f(x)= \begin{cases}-1 & \text { if } x<\frac{1}{2} \\ 1 & \text { if } x \geq \frac{1}{2}\end{cases}
$$

Then, $f \in \mathfrak{D}$. But

$$
\|f\|_{\mathfrak{D}}=\|f \wedge 0\|_{\mathfrak{D}}=1=\|f \vee 0\|_{\mathfrak{D}} .
$$

Thus,

$$
\|f \wedge 0\|_{\mathfrak{D}}+\|f \vee 0\|_{\mathfrak{D}}=2>1=\|f\|_{\mathfrak{D}}+\|0\|_{\mathfrak{D}} .
$$

Therefore, the inequality

$$
\|u \wedge v\|_{\mathfrak{D}}+\|u \vee v\|_{\mathfrak{D}} \leq\|u\|+\|v\|,
$$

does not hold for the energy norm of a Dirichlet form in general.

Theorem 4.11 Let $\mathcal{E}$ be a quasilinear, symmetric Dirichlet form. Then the lattice operations are continuous.

Proof At first, let $v \in \mathfrak{D}$ such that $v \geq 0$, let $\left(u_{n}\right)_{n \in \mathbb{N}}$ be a sequence in $\mathfrak{D}$ converging to 0 and $\lambda>0$. Then $u_{n} \wedge v \rightarrow 0$ and $u_{n} \vee v \rightarrow v$ in $L^{2}(X, m)$ for $n \rightarrow \infty$. Hence, the lower semicontinuity of $\mathcal{E}$ implies

$$
\begin{aligned}
\mathcal{E}_{1}(0)+\mathcal{E}_{1}(\lambda v) & \leq \liminf _{n \rightarrow \infty}\left(\mathcal{E}_{1}\left(\lambda\left(u_{n} \wedge v\right)\right)+\mathcal{E}_{1}\left(\lambda\left(u_{n} \vee v\right)\right)\right) \\
& \leq \limsup _{n \rightarrow \infty}\left(\mathcal{E}_{1}\left(\lambda\left(u_{n} \wedge v\right)\right)+\mathcal{E}_{1}\left(\lambda\left(u_{n} \vee v\right)\right)\right) \\
& \leq \limsup _{n \rightarrow \infty} \mathcal{E}_{1}\left(\lambda u_{n}\right)+\mathcal{E}_{1}(\lambda v),
\end{aligned}
$$

where we used lower semicontinuity and the first inequality from Definition 4.1. Since $\mathcal{E}_{1}$ is quasilinear, that is $\mathfrak{D}=\operatorname{dom} \mathcal{E}_{1}$, convex and lower semicontinuous on $\mathfrak{D}$, [17, Propsition 2.16] implies that $\mathcal{E}_{1}$ is continuous on $\mathfrak{D}$. Therefore, since $\mathcal{E}_{1}(0)=0$, the previous inequality yields

$$
\mathcal{E}_{1}(\lambda v)=\lim _{n \rightarrow \infty} \mathcal{E}_{1}\left(\lambda\left(u_{n} \wedge v\right)\right)+\mathcal{E}_{1}\left(\lambda\left(u_{n} \vee v\right)\right)
$$


We already know, that

$$
\mathcal{E}_{1}(\lambda v)=\mathcal{E}_{1}(\lambda(v \vee 0)) \leq \liminf _{n \rightarrow \infty} \mathcal{E}_{1}\left(\lambda\left(u_{n} \vee v\right)\right)
$$

Thus, $\lim _{n \rightarrow \infty} \mathcal{E}_{1}\left(\lambda\left(u_{n} \wedge v\right)\right)=0$ and Theorem 2.6 implies $u_{n} \wedge v \rightarrow 0$ in $\mathfrak{D}$. In addition, $v=v \wedge u_{n}+v \vee u_{n}-u_{n}$ which implies

$$
\lim _{n \rightarrow \infty} v \vee u_{n}=\lim _{n \rightarrow \infty} v+u_{n}-v \wedge u_{n}=v+0+0
$$

Hence, $v \vee u_{n} \rightarrow v \vee 0$.

Now, let $v \in \mathfrak{D}$ be arbitrary. Since $\left\|u_{n}^{+}\right\|_{\mathfrak{D}} \leq\left\|u_{n}\right\|_{\mathfrak{D}}$, we know that $u_{n}^{+}, u_{n}^{-} \rightarrow 0$. This implies

$$
v \wedge u_{n}=v^{+} \wedge u_{n}^{+}-v^{-} \vee u_{n}^{-} .
$$

We now use the first part again, which yields

$$
\begin{aligned}
\lim _{n \rightarrow \infty} v \wedge u_{n} & =\lim _{n \rightarrow \infty} v^{+} \wedge u_{n}^{+}-v^{-} \vee u_{n}^{-} \\
& =v^{+} \wedge 0-v^{-} \vee 0 \\
& =0+v \wedge 0 .
\end{aligned}
$$

Finally, let $\left(u_{n}\right)_{n}$ be an arbitrary sequence in $\mathfrak{D}$ converging to $u$. Then $u_{n}-u$ converges to 0 and

$$
\lim _{n \rightarrow \infty} u_{n} \wedge v=\lim _{n \rightarrow \infty}\left(u_{n}-u\right) \wedge(v-u)+u=0 \wedge(v-u)+u=u \wedge v .
$$

Hence, the infimum is separately continuous. Now, let $\left(u_{n}\right)_{n \in \mathbb{N}}$ and $\left(v_{n}\right)_{n \in \mathbb{N}}$ in $\mathfrak{D}$ converging to $u$ and $v$. Then

$$
v_{n} \wedge u_{n}=0 \wedge\left(u_{n}-v_{n}\right)+v_{n} .
$$

Hence,

$$
\lim _{n \rightarrow \mathbb{N}} v_{n} \wedge u_{n}=0 \wedge(u-v)+v=v \wedge u .
$$

The claim for the supremum follows by using the identity

$$
u+v=u \wedge v+u \vee v
$$

Lemma 4.12 If $\mathcal{E}$ is a symmetric Dirichlet form, then

$$
\mathcal{E}(-c \vee u \wedge c) \leq \mathcal{E}(u)
$$

for every $u \in \mathfrak{D}, c \geq 0$ and, if $\mathcal{E}$ is quasilinear, then

$$
\lim _{n \rightarrow \infty}-n \vee u \wedge n=u \text { in } \mathfrak{D} .
$$

Proof The map $p: \mathbb{R} \rightarrow \mathbb{R}$ given by $p(x)=(-n) \vee x \wedge n$ is a normal contraction. Hence, Theorem 4.7 implies

$$
\mathcal{E}_{1}(u-p(u-v))+\mathcal{E}_{1}(v+p(u-v)) \leq \mathcal{E}_{1}(u)+\mathcal{E}_{1}(v) .
$$

Setting $v=0$ implies

$$
\mathcal{E}_{1}((-n) \vee u \wedge n) \leq \mathcal{E}_{1}(u),
$$

since $\mathcal{E}_{1}(0)=0$ and $\mathcal{E}_{1} \geq 0$. 
For the second part, let us assume that $\mathcal{E}$ is quasilinear. By Property (3) in the definition of Dirichlet forms, we have

$$
\begin{aligned}
\mathcal{E}_{1}\left(v+\frac{1}{2}\left((u-v+\alpha)_{+}-(u-v-\alpha)_{-}\right)\right) \\
\quad+\mathcal{E}_{1}\left(u-\frac{1}{2}\left((u-v+\alpha)_{+}-(u-v-\alpha)_{-}\right)\right) \leq \mathcal{E}_{1}(u)+\mathcal{E}_{1}(v)
\end{aligned}
$$

for every $u, v \in L^{2}(X, m), \alpha>0$. Plugging in $v=0$ and replacing $\alpha$ by $n$ yields

$$
\mathcal{E}_{1}\left(\frac{1}{2}(u+(-n) \vee u \wedge n)\right)+\mathcal{E}_{1}\left(\frac{1}{2}(u-(-n) \vee u \wedge n)\right) \leq \mathcal{E}_{1}(u) .
$$

Let $\lambda>0$. Since $\lim _{n \rightarrow \infty}-n \vee u \wedge n=u$ in $L^{2}(X, m)$, the lower semicontinuity of $\mathcal{E}_{1}$ and the previous inequality imply

$$
\begin{aligned}
\mathcal{E}_{1}(0)+\mathcal{E}_{1}(\lambda u) & \leq \liminf _{n \rightarrow \infty} \mathcal{E}_{1}\left(\frac{\lambda}{2}(u+(-n) \vee u \wedge n)\right)+\mathcal{E}_{1}\left(\frac{\lambda}{2}(u-(-n) \vee u \wedge n)\right) \\
& \leq \limsup _{n \rightarrow \infty} \mathcal{E}_{1}\left(\frac{\lambda}{2}(u+(-n) \vee u \wedge n)\right)+\mathcal{E}_{1}\left(\frac{\lambda}{2}(u-(-n) \vee u \wedge n)\right) \\
& \leq \mathcal{E}_{1}(\lambda u),
\end{aligned}
$$

where we use the fact that for every $\lambda>0$ the functional $\mathcal{E}^{\lambda}(u):=\mathcal{E}(\lambda u)$ is also a symmetric, quasilinear Dirichlet form on $L^{2}(X, m)$. Since $\mathcal{E}_{1}(0)=0$,

$$
\lim _{n \rightarrow \infty} \mathcal{E}_{1}\left(\frac{\lambda}{2}(u+(-n) \vee u \wedge n)\right)+\mathcal{E}_{1}\left(\frac{\lambda}{2}(u-(-n) \vee u \wedge n)\right)=\mathcal{E}_{1}(\lambda u) .
$$

Since $\mathcal{E}_{1}$ is lower semicontinuous,

$$
\mathcal{E}_{1}(\lambda u) \leq \liminf _{n \rightarrow \infty} \mathcal{E}_{1}\left(\frac{\lambda}{2}(u+(-n) \vee u \wedge n)\right) .
$$

Since $\mathcal{E}_{1}$ is quasilinear, $\mathcal{E}_{1}(\lambda u)$ is finite and

$$
\begin{aligned}
0 \leq & \limsup _{n \rightarrow \infty} \mathcal{E}_{1}\left(\frac{\lambda}{2}(u-(-n) \vee u \wedge n)\right) \\
\leq & \limsup _{n \rightarrow \infty} \mathcal{E}_{1}\left(\frac{\lambda}{2}(u-(-n) \vee u \wedge n)\right)+\mathcal{E}_{1}\left(\frac{\lambda}{2}(u+(-n) \vee u \wedge n)\right) \\
& \quad+\limsup _{n \rightarrow \infty}-\mathcal{E}_{1}\left(\frac{\lambda}{2}(u+(-n) \vee u \wedge n)\right) \\
\leq & \mathcal{E}_{1}(\lambda u)-\mathcal{E}_{1}(\lambda u) \\
= & 0
\end{aligned}
$$

Hence,

$$
\lim _{n \rightarrow \infty} \mathcal{E}_{1}\left(\frac{\lambda}{2}(u-(-n) \vee u \wedge n)\right)=0 .
$$

Theorem 2.6 implies $\frac{1}{2}(u-(-n) \vee u \wedge n) \rightarrow 0$ for $n \rightarrow \infty$ in $\mathfrak{D}$. Since $u$ was arbitrary, this yields $(-n) \vee u \wedge n \rightarrow u$ in $\mathfrak{D}$ for every $u \in \mathfrak{D}$ and $n \rightarrow \infty$.

Corollary 4.13 Let $u, g \in \mathfrak{D}$. Then $u \wedge(c-g) \in \mathfrak{D}$ for every $c>0$. 
Proof

$$
u \wedge(c-g)=((u+g) \wedge c)-g
$$

We give an example of a Dirichlet form where the lattice operations are not continuous on the associated Dirichlet space.

Example 4.14 Let $X=[0,1]$ with the usual Lebesgue measure. Let

$$
\mathcal{E}(u)= \begin{cases}0 & \text { if } u^{\prime} \text { exists and }\left|u^{\prime}\right| \leq 1 \text { a.e. } \\ \infty & \text { else. }\end{cases}
$$

Let us show that $\mathcal{E}$ is lower semicontinuous. Let $\left(u_{n}\right)_{n \in \mathbb{N}}$ be a sequence in $L^{2}(X, m)$ converging to $u$ such that $\mathcal{E}\left(u_{n}\right)=0$ for every $n \in \mathbb{N}$. Then, $\left\|u_{n}^{\prime}\right\|_{L^{\infty}} \leq 1$ and there exists $v \in L^{\infty}(X),\|v\|_{\infty} \leq 1$ such that, up to a subsequence, $u_{n}^{\prime} \rightarrow v$ in the weak* topology of $L^{\infty}(X)$. For every $\phi \in C_{c}^{\infty}(X)$

$$
\int_{X} u_{n}^{\prime} \phi=-\int_{X} u_{n} \phi^{\prime}
$$

Passing to the limit yields

$$
\int_{X} v \phi=-\int_{X} u \phi^{\prime}
$$

Therefore $u^{\prime}=v$ and $\mathcal{E}(u)=0$.

It is easy to see that $\mathcal{E}$ is a Dirichlet form. The Dirichlet space $\mathfrak{D}$ coincides with $W^{1, \infty}(X)$. Let $f(x)=x$ and $g_{n}(x)=\frac{1}{n}$. Then $g_{n} \rightarrow 0$ in $\mathfrak{D}$ but

$$
\left\|f \wedge g_{n}\right\|_{\mathfrak{D}} \geq 1
$$

for every $n \in \mathbb{N}$. Hence, $f \wedge g_{n}$ does not converge to 0 in $\mathfrak{D}$.

\section{Capacity}

One tool in the study of classical Dirichlet forms is the capacity. For a reference in the classical case, see for example [8]. From now on let us assume that $X$ is a topological measure space and supp $m=X$.

In this section $\mathcal{E}$ denotes a symmetric Dirichlet form and $\left(\mathfrak{D},\|\cdot\|_{\mathfrak{D}}\right)$ the associated Dirichlet space.

Definition 5.1 Let $A \subseteq X$. We define the set

$\mathcal{L}_{A}=\left\{u \in L^{2}(X, m) \mid\right.$ there exists an open set $U$ such that $A \subseteq U$ and $u \geq 1$ a.e. on $\left.U\right\}$, and the (norm-) capacity by

$$
\operatorname{Cap}_{\mathfrak{D}}(A)=\inf \left\{\|u\|_{\mathfrak{D}} \mid u \in \mathcal{L}_{A}\right\} .
$$

Lemma 5.2 Let $A, B \subseteq X$. Then $\operatorname{Cap}_{\mathfrak{D}}(A \cup B) \leq \operatorname{Cap}_{\mathfrak{D}}(A)+\operatorname{Cap}_{\mathfrak{D}}(B)$ and if $A \subseteq B$, then $\operatorname{Cap}_{\mathfrak{D}}(A) \leq \operatorname{Cap}_{\mathfrak{D}}(B)$. 
Proof For the first part, let us consider two arbitrary functions $f_{A} \in \mathcal{L}_{A}, f_{B} \in \mathcal{L}_{B}$. Then, by Theorem 4.9 ,

$$
\operatorname{Cap}_{\mathfrak{D}}(A \cup B) \leq\left\|f_{A} \vee f_{B}\right\|_{\mathfrak{D}} \leq\left\|f_{A}\right\|_{\mathfrak{D}}+\left\|f_{B}\right\|_{\mathfrak{D}} .
$$

Since $f_{A}, f_{B}$ are arbitrary, this implies the first claim. The second part follows directly from the definition and properties of the infimum.

Lemma 5.3 Let $A_{n}, A \subseteq X$ such that $\bigcup_{n=1}^{\infty} A_{n}=A$. Then $\sum_{n=1}^{\infty} \operatorname{Cap}_{\mathfrak{D}}\left(A_{n}\right) \geq \operatorname{Cap}_{\mathfrak{D}}(A)$.

Proof Let $A_{n}, A \subseteq X$ as above.

Let us assume $\sum_{n=1}^{\infty} \operatorname{Cap}_{\mathfrak{D}}\left(A_{n}\right)<\infty$. Otherwise there is nothing to show. Let $\epsilon>0$. By the properties of the infimum, we can choose functions $u_{n} \in L^{2}(X, m)$ and open sets $U_{n}$ for every $n \in \mathbb{N}$ such that $u_{n} \geq 1$ almost everywhere on $U_{n}$ and $A_{n} \subseteq U_{n}$

$$
\left\|u_{n}\right\|_{\mathfrak{D}} \leq \operatorname{Cap}_{\mathfrak{D}}\left(A_{n}\right)+\epsilon 2^{-n} .
$$

Without loss of generality we assume $u_{n} \leq 1$, otherwise take $u_{n} \wedge 1$. Set

$$
U=\bigcup_{n=1}^{\infty} U_{n}
$$

and

$$
g_{n}=\bigvee_{i=1}^{n} u_{i}
$$

By Theorem 4.9,

$$
\left\|g_{n}\right\|_{\mathfrak{D}} \leq \sum_{n=1}^{\infty} \operatorname{Cap}_{\mathfrak{D}}\left(A_{n}\right)+\epsilon<\infty .
$$

Since $\|\cdot\|_{L^{2}(X, m)} \leq\|\cdot\|_{\mathfrak{D}}$, the sequence $g_{n}$ is bounded in $L^{2}$. In addition $\left(g_{n}\right)_{n \in \mathbb{N}}$ is pointwise increasing. Thus, by the monotone convergence theorem, there exists a $g \in L^{2}(X, m)$ such that $g_{n} \rightarrow g$ in $L^{2}(X, m)$ and pointwise almost everywhere. Hence, $g=1$ almost everywhere on $U_{k}$ for every $k \in \mathbb{N}$. Thus, $g=1$ a.e. on $U$ and, by the weak lower semicontinuity of $\|\cdot\|_{\mathfrak{D}}$ on $L^{2}(X, m)$, we have

$$
\begin{aligned}
\operatorname{Cap}_{\mathfrak{D}}(A) & \leq\|g\|_{\mathfrak{D}} \\
& \leq \liminf _{n \rightarrow \infty}\left\|g_{n}\right\|_{\mathfrak{D}} \\
& \leq \sum_{n=1}^{\infty} \operatorname{Cap}_{\mathfrak{D}}\left(A_{n}\right)+\epsilon .
\end{aligned}
$$

Since $\epsilon$ was arbitrary, this concludes the proof.

Lemma 5.4 Let $K_{n} \subseteq X$ be compact subsets such that $K_{n} \downarrow K$. Then

$$
\operatorname{Cap}_{\mathfrak{D}}(K)=\inf _{n \in \mathbb{N}} \operatorname{Cap}_{\mathfrak{D}}\left(K_{n}\right) .
$$

Proof By the monotonicity of $\mathrm{Cap}_{\mathfrak{D}}, \mathrm{Cap}_{\mathfrak{D}}(K) \leq \inf _{n \in \mathbb{N}} \mathrm{Cap}_{\mathfrak{D}}\left(K_{n}\right)$. 
For the converse inequality, let us choose an $\epsilon>0$, an open neighborhood $U$ of $K$ and a function $f \in \mathcal{L}_{U}$ such that

$$
\|f\|_{\mathfrak{D}} \leq \operatorname{Cap}_{\mathfrak{D}}(K)+\epsilon
$$

We know

$$
\emptyset=K \cap U^{c}=\bigcap_{i=1}^{\infty} K_{i} \cap\left(U^{c} \cap K_{1}\right) .
$$

For every $i \in \mathbb{N}, K_{i} \subseteq K_{1}$ and $U^{c} \cap K_{1} \subseteq K_{1}$. Since $K_{1}$ is compact, we can apply the finite intersection property. Hence, there are finitely many $K_{i_{1}}, \ldots, K_{i_{n}}$ such that

$$
\emptyset=\bigcap_{k=1}^{n} K_{i_{k}} \cap\left(U^{c} \cap K_{1}\right)=K_{i_{n}} \cap\left(U^{c} \cap K_{1}\right)=K_{i_{n}} \cap U^{c} .
$$

Thus, $U$ is an open neighborhood of $K_{i_{n}}$ and, by the definition of the capacity,

$$
\inf _{n \in \mathbb{N}} \operatorname{Cap}_{\mathfrak{D}}\left(K_{n}\right) \leq \operatorname{Cap}_{\mathfrak{D}}\left(K_{i_{n}}\right) \leq\|f\|_{\mathfrak{D}} \leq \operatorname{Cap}_{\mathfrak{D}}(K)+\epsilon .
$$

Since $\epsilon$ is arbitrary, the claim follows.

Definition 5.5 We call a set $A \subseteq X$ polar, if $\operatorname{Cap}_{\mathfrak{D}}(A)=0$, and some property holds quasi everywhere (q.e.), if it holds up to a polar set.

Example 5.6 Let $X \subseteq \mathbb{R}^{n}$ a bounded domain, $m$ the Lebesgue measure and $p \geq 1$. Then,

$$
\mathcal{E}(u)=\frac{1}{p} \int_{X}|\nabla u|^{p}
$$

is a convex, lower semicontinuous Dirichlet form. The Dirichlet space $\mathfrak{D}=W_{p, 2}^{1}(X)=$ $\left\{u \in L^{2}(X, m) \mid \nabla u \in L^{p}\right\}$ and the capacity of $\mathcal{E}$ is the relative $p$-capacity (or the usual $p$-capacity if $\left.X=\mathbb{R}^{n}\right)$. Let $0<\alpha \leq 2$. The perturbation

$$
\mathcal{E}_{\alpha}(u)=\mathcal{E}(u)+\int_{X}|u|^{\alpha}
$$

is also a convex, lower semicontinuous Dirichlet form.

Let $A \subseteq X$ be a Cap $\mathcal{D E}_{\mathcal{E}}$-polar set. Then there is a sequence $\left(u_{n}\right)_{n \in \mathbb{N}}$ in $\mathfrak{D}$ such that $u_{n}=$ 1 on a neighbourhood of $A$ and $u_{n} \rightarrow 0$ in $\mathfrak{D}$. Hence, $u_{n} \rightarrow 0$ in $L^{2}(X, m)$. By the Hölder inequality $u_{n} \rightarrow 0$ in $L^{\alpha}(X)$. Therefore $A$ is $\operatorname{Cap}_{\mathfrak{D}} \mathcal{E}_{\alpha}$-polar. The converse implication, namely that every $\operatorname{Cap}_{\mathfrak{D} \mathcal{E}_{\alpha}}$-polar set is $\mathrm{Cap}_{\mathfrak{D} \mathcal{E}}$-polar, is clear. Hence, the capacities possess the same polar sets. Note carefully that the corresponding Dirichlet spaces need not be the same.

\section{Quasicontinuity}

In this section $\mathcal{E}$ denotes a symmetric Dirichlet form.

Definition 6.1 We say $f: X \rightarrow Y$ for some topological space $Y$ is quasicontinuous, if for every $\epsilon>0$ there is an open set $O \subseteq X$ such that $\operatorname{Cap}_{\mathfrak{D}}(O) \leq \epsilon$ and $\left.f\right|_{O^{c}}$ is continuous.

Additionally we call $f \in L^{2}(X, m)$ quasicontinuous, if there is a representative which is quasicontinuous. Whenever this is the case, we denote this representative again by $f$. 
Lemma 6.2 Let $A \subseteq X$ be measurable and polar. Then $m(A)=0$.

Proof Let $A \subseteq X$ be measurable and polar. Then there exists a sequence of open sets $A \subseteq O_{n} \subseteq X$ and functions $f_{n} \geq 0$ such that $f_{n} \geq 1$ a.e. on $O_{n}$ and $\left\|f_{n}\right\|_{\mathfrak{D}} \leq \frac{1}{n}$. Hence,

$$
m(A)=\left\|1_{A}\right\|_{2}^{2} \leq\left\|f_{n}\right\|_{2}^{2} \leq\left\|f_{n}\right\|_{\mathfrak{D}}^{2} \leq \frac{1}{n^{2}},
$$

which implies $m(A)=0$.

Theorem 6.3 Let $U \subseteq X$ be an open subset and $f: X \rightarrow \mathbb{R}$ quasicontinuous. Then

$$
f \geq 0 \text { a.e. on } U \Longleftrightarrow f \geq 0 \text { q.e. on } U
$$

Proof Let us assume $f \geq 0$ a.e. on U. We choose an $\epsilon>0$ and an open set $O \subseteq X$ as in the definition of quasicontinuity. We know that $N=\{f<0\} \cap U$ has measure 0 . First observe that $f$ is continuous on $O^{c}$ and thus $O^{c} \cap\{f<0\}$ is open in $O^{c}$. This shows that

$$
O^{\prime}=O \cup N=O \cup\left((N \cap O) \cup\left(N \cap O^{c}\right)\right)=O \cup\left(N \cap O^{c}\right)
$$

is open in $X$ by the definition of the induced topology. Thus, $\operatorname{Cap}_{\mathfrak{D}}\left(O^{\prime}\right)=\operatorname{Cap}_{\mathfrak{D}}(O)$, since $\mathcal{L}_{O}=\mathcal{L}_{O^{\prime}}$. Therefore

$$
\operatorname{Cap}_{\mathfrak{D}}(N) \leq \operatorname{Cap}_{\mathfrak{D}}\left(O^{\prime}\right)=\operatorname{Cap}_{\mathfrak{D}}(O)<\epsilon .
$$

Since $\epsilon$ was arbitrary we have $\operatorname{Cap}_{\mathfrak{D}}(N)=0$. The other direction follows directly from Lemma 6.2.

Corollary 6.4 Let $f_{1}, f_{2}: X \rightarrow \mathbb{R}$ be two quasicontinuous representatives of some $f \in$ $L^{2}(X, m)$. Then $f_{1}=f_{2}$ quasi everywhere on $X$.

Definition 6.5 We call a set $U \subseteq X$ quasi open if, for every $\epsilon>0$ there is an open set $O_{\epsilon} \subseteq X$ such that $U \subseteq O_{\epsilon}$ and $\operatorname{Cap}_{\mathfrak{D}}\left(O_{\epsilon} \backslash U\right) \leq \epsilon$.

Lemma 6.6 A function $f \in L^{2}(X, m)$ is quasicontinuous if and only if, for every open set $U \subseteq \mathbb{R}, f^{-1}(U)$ is quasi open.

Proof The proof works exactly like the one in the bilinear case [8].

Theorem 6.7 Let $f \in \mathfrak{D}$ be a quasicontinuous function and $\lambda>0$. Then

$$
\operatorname{Cap}_{\mathfrak{D}}(\{|f|>\lambda\}) \leq \lambda^{-1}\|f\|_{\mathfrak{D}} .
$$

Proof Let $\lambda>0$ and $\epsilon>0$. Since $f$ is quasicontinuous, there is an open set $U_{\epsilon}$ with $\operatorname{Cap}_{\mathfrak{D}}\left(U_{\epsilon}\right) \leq \epsilon$ and $f$ is continuous on $U_{\epsilon}^{c}$. There is a function $g_{\epsilon}$, such that $g_{\epsilon} \geq 1$ on $U_{\epsilon}$ and $\left\|g_{\epsilon}\right\|_{\mathfrak{D}} \leq 2 \epsilon$. Additionally, the set $O=\{|f|>\lambda\} \cup U_{\epsilon}$ is open. Note that $\left(\lambda^{-1}|f|\right) \vee g_{\epsilon} \geq 1$ on $O$. Hence, by Remark 4.4s and Theorem 4.9,

$$
\begin{aligned}
\operatorname{Cap}_{\mathfrak{D}}(\{|f|>\lambda\}) & \leq \operatorname{Cap}_{\mathfrak{D}}(O) \leq\left\|\left(\lambda^{-1}|f|\right) \vee g_{\epsilon}\right\|_{\mathfrak{D}} \\
& \leq\left\|\lambda^{-1}|f|\right\|_{\mathfrak{D}}+\left\|g_{\epsilon}\right\|_{\mathfrak{D}} \leq \lambda^{-1}\|f\|_{\mathfrak{D}}+2 \epsilon .
\end{aligned}
$$

Since $\epsilon$ is arbitrary, this implies the claim. 
Theorem 6.8 Let $\left(f_{n}\right)_{n}$ be a sequence of quasicontinuous functions in $\mathfrak{D}$ and $f \in \mathfrak{D}$ with $f_{n} \rightarrow f$ in $\mathfrak{D}$. Then $f$ is quasicontinuous and there exists a subsequence which converges pointwise quasi everywhere and quasi uniformly, that is for every $\epsilon>0$ there is a open set $U \subseteq X$ such that $\mathrm{Cap}_{\mathfrak{D}}(U) \leq \epsilon$ and $f_{n}$ converges uniformly to $f$ on $U^{c}$.

Proof Let us choose a subsequence $\left(f_{n}\right)_{n}$ such that

$$
\left\|\left|f_{n}-f_{n+1}\right|\right\|_{\mathfrak{D}} \leq\left\|f_{n}-f_{n+1}\right\|_{\mathfrak{D}} \leq 2^{-2 n}
$$

Then, since $\left|f_{n}-f_{n+1}\right|$ is quasicontinuous, Theorem 6.7 yields

$$
\operatorname{Cap}_{\mathfrak{D}}\left(\left\{\left|f_{n}-f_{n+1}\right|>2^{-n}\right\}\right) \leq 2^{-n} .
$$

Hence, for every $m \in \mathbb{N}$ we have

$$
\operatorname{Cap}_{\mathfrak{D}}\left(\bigcup_{n \geq m}\left\{\left|f_{n}-f_{n+1}\right|>2^{-n}\right\}\right) \leq 2^{-m+1} .
$$

In addition, let $\epsilon>0$. Since $f_{n}$ is quasicontinuous, we can choose an open set $U_{n}$ such that

$$
\operatorname{Cap}_{\mathfrak{D}}\left(U_{n}\right) \leq \epsilon 2^{-n}
$$

and $\left.f_{n}\right|_{U_{n}^{c}}$ is continuous. Set $U:=\bigcup_{n=1}^{\infty} U_{n}$. Then

$$
\operatorname{Cap}_{\mathfrak{D}}(U)=\operatorname{Cap}_{\mathfrak{D}}\left(\bigcup U_{n}\right) \leq \epsilon .
$$

Therefore, the sequence $\left(\left.f_{n}\right|_{U^{c}}\right)_{n \in \mathbb{N}}$ of continuous functions converges uniformly on $A_{m}=$ $\bigcap_{n \geq m}\left\{\left|f_{n}-f\right| \leq 2^{-n}\right\}$. Thus, $\left.f\right|_{U^{c} \cap A_{m}}$ is continuous, since it is the uniform limit of continuous functions, and

$$
\begin{aligned}
\operatorname{Cap}_{\mathfrak{D}}\left(\left(A_{m} \cap U^{c}\right)^{c}\right) & \leq \operatorname{Cap}_{\mathfrak{D}}\left(\bigcup_{n \geq m}\left\{\left|f_{n}-f_{n+1}\right|>2^{-n}\right\}\right)+\operatorname{Cap}_{\mathfrak{D}}(U) \\
& \leq 2^{-m+1}+\epsilon .
\end{aligned}
$$

This implies, that $f$ is quasicontinuous. Next, we show that a subsequence of $f_{n}$ converges pointwise quasi everywhere. For this purpose, let us choose a different subsequence such that

$$
\left\|\left|f_{n}-f\right|\right\|_{\mathfrak{D}} \leq\left\|f_{n}-f\right\|_{\mathfrak{D}} \leq 2^{-2 n} .
$$

Let $m \in \mathbb{N}$ be arbitrary and $x \in \bigcap_{n \geq m}\left\{\left|f_{n}-f\right| \leq 2^{-n}\right\}$. Then $f_{n}(x) \rightarrow f(x)$. Thus, $A=\left\{x \mid f_{n}(x) \nrightarrow f(x)\right\}$ is a subset of $\bigcup_{n \geq m}\left\{\left|f_{n}-f\right|>2^{-n}\right\}$ for every $m$. The Chebychev type inequality Theorem 6.7 yields

$$
\operatorname{Cap}_{\mathfrak{D}}(A) \leq \operatorname{Cap}_{\mathfrak{D}}\left(\bigcup_{n \geq m}\left\{\left|f_{n}-f\right|>2^{-n}\right\}\right) \leq 2^{-m+1}
$$

for every $m$. Therefore

$$
\operatorname{Cap}_{\mathfrak{D}}(A)=0 .
$$

Corollary 6.9 Let $f \in \overline{\mathfrak{D} \cap C(X)}^{\mathfrak{D}}$. Then $f$ is quasicontinuous on $X$. 
Proof Every continuous function is quasicontinuous. Hence, the previous theorem implies the claim.

Remark 6.10 For $\mathcal{E}=\|\cdot\|_{2}$ this theorem is a version of Lusin's Theorem and Egorov's theorem. Furthermore, Theorem 6.7 is a version of the Markov or weak $L^{1}$ inequality.

Acknowledgements author would like to thank the referee for the careful reading of the manuscript and the valuable suggestions, which have been very helpful in improving the paper.

Funding Open Access funding enabled and organized by Projekt DEAL.

Open Access This article is licensed under a Creative Commons Attribution 4.0 International License, which permits use, sharing, adaptation, distribution and reproduction in any medium or format, as long as you give appropriate credit to the original author(s) and the source, provide a link to the Creative Commons licence, and indicate if changes were made. The images or other third party material in this article are included in the article's Creative Commons licence, unless indicated otherwise in a credit line to the material. If material is not included in the article's Creative Commons licence and your intended use is not permitted by statutory regulation or exceeds the permitted use, you will need to obtain permission directly from the copyright holder. To view a copy of this licence, visit http://creativecommons.org/licenses/by/4.0/.

\section{References}

1. Rockafellar, R.T.: The theory of subgradients and its applications to problems of optimization, volume 1 of R \& E. Heldermann, Berlin. Convex and nonconvex functions (1981)

2. Lions, J.-L.: Quelques méthodes de résolution des problèmes aux limites non linéaires. Dunod, GauthierVillars, Paris (1969)

3. Brezis, H.: Opérateurs maximaux monotones et semi-groupes de contractions dans les espaces de Hilbert. North-Holland Publishing Co., Amsterdam-London; American Elsevier Publishing Co., Inc., New York. North-Holland Mathematics Studies, No. 5. Notas de Matemática (50) (1973)

4. Chill, R., Mildner, S.: The Kurdyka-łojasiewicz-Simon inequality and stabilisation in nonsmooth infinite-dimensional gradient systems. Proc. Amer. Math. Soc. 146(10), 4307-4314 (2018)

5. Cipriani, F., Grillo, G.: Nonlinear Markov semigroups, nonlinear Dirichlet forms and applications to minimal surfaces. J. Reine Angewandte Math. (Crelle's J.) 562, 201-235 (2003)

6. Barthélemy, L.: Invariance d'un convexe fermé par un semi-groupe associé à une forme non-linéaire. Abstr. Appl. Anal. 1(3), 237-262 (1996)

7. Bénilan, P., Crandall, M.G.: Completely accretive operators. In: Semigroup theory and evolution equations (Delft, 1989), Lecture Notes in Pure and Appl. Math., vol. 135, pp. 41-75. Dekker, New York (1991)

8. Fukushima, M., Oshima, Y., Takeda, M.: Dirichlet forms and symmetric Markov processes, De Gruyter Studies in Mathematics, vol. 19. Walter de Gruyter \& Co., Berlin. extended edition (2011)

9. Bouleau, N., Hirsch, F.: Dirichlet forms and analysis on Wiener space, De Gruyter Studies in Mathematics, vol. 14. Walter de Gruyter \& Co., Berlin (1991)

10. Ma, Z.M., Röckner, M.: Introduction to the theory of (nonsymmetric) Dirichlet forms. Universitext. Springer, Berlin (1992)

11. Biroli, M.: Strongly local nonlinear Dirichlet functionals. Ukr. Mat. Visn. 1(4), 485-500 (2004)

12. Biroli, M., Vernole, P.G.: Strongly local nonlinear Dirichlet functionals and forms. Adv. Math. Sci. Appl. 15(2), 655-682 (2005)

13. Musielak, J.: Orlicz spaces and modular spaces, Lecture Notes in Mathematics, vol. 1034. Springer, Berlin (1983)

14. Diening, L., Harjulehto, P., Hästö, P., Ruzicka, M.: Lebesgue and sobolev spaces with variable exponents, Lecture Notes in Mathematics, vol. 2017. Springer, Berlin (2011)

15. Kaijser, S.: A note on dual Banach spaces. Math. Scand. 41(2), 325-330 (1977) 
16. Bénilan, P., Picard, C.: Quelques aspects non linéaires du principe du maximum. In: Séminaire de Théorie du Potentiel, No. 4 (Paris, 1977/1978), Lecture Notes in Math., vol. 713, pp. 1-37. Springer, Berlin (1979)

17. Barbu, V., Precupanu, T.: Convexity and optimization in Banach spaces, 4th edn. Springer Monographs in Mathematics. Springer, Dordrecht (2012)

Publisher's Note Springer Nature remains neutral with regard to jurisdictional claims in published maps and institutional affiliations. 